\title{
Impact of a Double Epidemic in Mexico: Non- Communicable Diseases Increase the Case Fatality Rate with Covid-19
}

\section{Eric Monterrubio-Flores}

Instituto Nacional de Salud Publica

Maria Dolores Ramírez-Villalobos ( $\nabla$ dolores.ramirez@insp.mx )

Instituto Nacional de Salud Publica https://orcid.org/0000-0002-0625-4648

Juan Espinoza-Montero

Instituto Nacional de Salud Publica

\section{Bernardo Hernandez}

Institute for Health Metrics and Evaluation

\section{Simón Barquera}

Instituto Nacional de Salud Publica

Victor E. Villalobos-Daniel

Berkeley University

\section{Ismael Campos-Nonato}

Instituto Nacional de Salud Publica

Original investigation

Keywords: hypertension, diabetes, cardiovascular disease, COVID-19, mortality

Posted Date: September 25th, 2020

DOl: https://doi.org/10.21203/rs.3.rs-80669/v1

License: (c) (1) This work is licensed under a Creative Commons Attribution 4.0 International License. Read Full License 


\section{Abstract}

Background: People with a previous diagnosis of non-communicable diseases (NCDs) develop serious forms of COVID-19 or die. Mexico is the sixth country with the highest mortality rate by COVID-19 with high mortality in younger adults.

Objectives: To describe the association between NCDs and Case Fatality Rate (CFR) due to COVID-19 and identify related factors.

Methods: We studied all Mexican adults infected with COVID-19 from February 1st to July 31st, 2020. The data set is available for free on the Ministry of Health's webpage. We analyzed previous diagnosis of NCDs, other preexisting diseases, indicators of severity, and the institution treating the patient. We estimated CFR and analyzed it by the number of NCDs, specific diseases, and combinations of NCDs. Relative risk was estimated using Poisson models and probability of death estimates with Logistic models.

Results: We analyzed 406,966 COVID-19 positive adults. The general CFR was $11.2 \%$, while for men it was $13.7 \%$ and for women $8.4 \%$. CFR is positively associated with age and number of NCDs (trend test $\mathrm{p}<0.001)$. The combination of type 2 diabetes+chronic kidney failure has the highest CFR (44.0\%). The number of comorbidities increase the CFR in younger adults, in which the relative risk from 0 to 3 or more NCDs was 30.2 in women and 11.8 in men. We observed great heterogeneity in the CFR by institution, from $4.6 \%$ in private institutions to $18.9 \%$ in public institutions.

Conclusion: The number of NCDs increases CFR and explains the mortality in younger adults. Our findings are consistent with the scientific literature and contribute to the understanding of these associations.

\section{Background}

The severe acute respiratory syndrome coronavirus 2 disease (COVID-19) ${ }^{1}$ can produce mild respiratory symptoms that remit naturally, ${ }^{2}$ but in some cases can also evolve to acute respiratory distress syndrome, causing multiple organ failure and death. ${ }^{3}$

As of July 31 st, 2020 , more than 17.3 million people have been infected with COVID-19 worldwide and more than 674,000 have died from this cause. When comparing the mortality rate per 100,000 inhabitants during this period, Mexico was six country with the highest mortality rate. Mexico had officially tested 984,288 people with 424,637 positive cases. ${ }^{4}$

People with a previous diagnosis of non-communicable diseases (NCDs) such as high blood pressure (HBP), type 2 diabetes (T2D), cardiovascular disease (CVD), and chronic obstructive pulmonary disease

(COPD) ${ }^{5-6}$ as well as those who are older or men, are more likely to develop serious conditions of COVID19 or die from this cause. ${ }^{7}$ In China, the fatality rate (number of deaths per 100 infected) for COVID-19 
was $2.3 \%$. However, it was $6.0 \%$ for people with hypertension, $7.3 \%$ for adults with diabetes and $10.5 \%$ for people with cardiovascular disease $10.5 \%{ }^{8}$ On the other hand, those who developed severe symptoms or who died in a hospital were mostly men or people over the age of 65 , with a hazard risk (HR) of 1.9 and 1.6 respectively. ${ }^{9}$ Epidemiological studies have shown that the risk of mortality from COVID-19 increases 2.5 times when the patient has high blood pressure, 1.9 times when they have diabetes ${ }^{10}$ and 7.9 times when they have CVD. ${ }^{11}$

In Mexico, the association between obesity and diabetes with a higher risk of COVID-19 14 infection, severity and need of hospitalization has been documented. ${ }^{13} \mathrm{~A}$ recent study has also documented a higher risk of complications at the beginning of hospitalization among patients with COVID-19 who also had comorbidities like obesity, hypertension and diabetes. ${ }^{15}$

It has been shown that NCDs increase the severity from COVID-19. In a country like Mexico where $49 \%$ of adults have hypertension, ${ }^{16} 14 \%$ have diabetes, ${ }^{17}$ and $24 \%$ develop CVD, ${ }^{18}$ it is important to quantify the risk of death among the population with NCDs and COVID-19. It is also important to consider the role of the health system providing care to patients with COVID-19 in cases with and without other NCDs. Understanding the magnitude of this association and its related characteristics can improve targeted strategies that aim to identify adults who are most likely to be infected and die of COVID-19. In the present study, we aim to analyze if adults in Mexico with NCDs have an increased risk of death when infected with COVID-19, and if this risk is also associated with their sex.

\section{Methods}

\section{Study design and participants}

We conducted a study population consisted of Mexican adults aged 20 years older who tested positive for COVID-19, registered in the Epidemiological Surveillance System for Respiratory Diseases database (SISVER, Spanish acronym). This database includes epidemiological information at the national level, with mandatory reporting of diseases like COVID-19 for all public or private health units and laboratories. The information was obtained from the Ministry of Health's (https://www.gob.mx/salud/documentos/datos-abiertos-152127). This is public information that can be accessed for free by any user. ${ }^{19}$ This dataset is continuously updated, and we analyzed data from the beginning of the epidemic (February 28,2020 ) to July 31,2020 . The database contains information on all outpatients and those hospitalized, as well as on deaths from COVID-19 and cases diagnosed through the sentinel surveillance system. ${ }^{19}$

\section{Confirmation of cases with COVID-19}

An adult was considered to have COVID-19 when reporting to have at least two of the following symptoms in the last 7 days: fever, headache and cough, accompanied by at least one of the following signs: arthralgia, conjunctivitis, pain in the chest, dyspnea, myalgia, odynophagia or rhinorrhea. For all 
suspected cases, two protocols were followed: testing for COVID-19 and epidemiological surveillance. ${ }^{19}$ As authorized by the National Committee for Epidemiological Surveillance (CONAVE, Spanish acronym),${ }^{19}$ cases were confirmed using the polymerase chain reaction (PCR) test based on the Berlin protocol. ${ }^{20}$

\section{NCDs Assessment}

An adult was considered to have an NCDs when the patient reported been diagnosed by with: HBP, type 2 diabetes (T2D), obesity (OB), CVD, chronic kidney failure (CKD) or COPD. It was also considered as having these conditions if the patient self-reported having NDCs or if their medical history described it. Using a standardized questionnaire (study format of a suspected case of respiratory disease caused by virus), ${ }^{19}$ getting the: sociodemographic information (age and sex), personal pathological history (presence of $\mathrm{NCDs}$, date on which the symptoms associated with the infection began, and exposure to tobacco), treatment characteristics, and indicators of severity such as the presence of pneumonia, need for hospitalization, assistance in the Intensive Care Unit (ICU), and the use of assisted mechanical intubation (IMA). We classified information on non-communicable diseases by number, as follows: No NCDs, one NCDs, two NCDs, and three or more NCDs.

Institution where health care was provided

We included in the analysis the institution where patients received care. For the analysis, we categorized the health care institutions as Private, Ministry of Navy (SEMAR), Federal Ministry of Health (SS), (Red Cross + DIF + Municipal + Universitary), Ministry of National Defense (SEDENA), not specified, State Secretaries of Health, Petroleos Mexicanos (PEMEX), Institute of Security and Social Services for State Workers (ISSSTE), and Mexican Institute of Social Security (IMSS).

\section{Statistical analysis}

The fatality due to COVID-19 was calculated through Case Fatality Rate (CFR) and expressed in percentages (number of deaths from COVID / total patients identified with COVID in the period described $X 100)$ with their $95 \%$ confidence intervals $(95 \% \mathrm{Cl})$.

The CFR was disaggregated by sex, age, and NCDs. To assess trends between age groups and number of NCDs, tests were performed using robust estimates by Bootstrap. The relative risk (RR) was estimated using Poisson models and the probability of death (CFR adjusted) using logistic regression, which in both cases were adjusted for age, sex, the presence of pre-existing diseases (asthma, immunosuppression, other comorbidities non-specified) and Institutions of the Health System, including them as dummy variables in the models. Robust estimates were used in all models. Data analysis was performed using the statistical software STATA, version 14 (College Station, TX, EE. UU.) and the statistical software R (R Core Team 2020) R (A language and environment for statistical computing. R Foundation for Statistical Computing, Vienna, Austria. URL https://www.r-project.org/). 


\section{Ethical considerations}

The study does not require ethical review because it is based on open, anonymized data from the Mexican Ministry of Health.

\section{Results}

The database under study included information on 842,025 adults aged 20 years or older with a test of for COVID-19. Of these, 3,600 (0.4\%) observations were eliminated for not having information related to the diagnosis of NCDs. In this study we analyzed information only from cases with confirmed COVID-19 $(n=406,966)$. Out of them, $53.2 \%$ were men and $46.5 \%$ women). Women had a mean age of 45.6 years $(95 \% \mathrm{Cl} 45.6,45.7)$ and men 47.0 years $(95 \% \mathrm{Cl} 46.9,47.1)$.

Participants' characteristics by sex are described in Table 1. The prevalence of NCDs was similar between sex. The most common NCD was HBP $(20.6 \%, 95 \% \mathrm{Cl} 20.5,20.8)$, followed by obesity $(19.8 \%, 95 \% \mathrm{Cl}$ $19.6,19.8)$ and diabetes $(16.8 \%, 95 \% \mathrm{Cl} 16.7 \%, 16.9)$. Furthermore, $55.8 \%(95 \% \mathrm{Cl} 55.6,56.0)$ reported not having any NCDs, while $25.9 \%(95 \% \mathrm{Cl} 25.8,26.1)$ reported having one, $12.1 \%(95 \% \mathrm{Cl} 12.0,12.3)$ reported having two, and $5.9 \%(95 \% \mathrm{Cl} 5.9,6.0)$ had three or more. Over half of the cases were reported by units of the Secretary of Health $(53.7 \%(95 \% \mathrm{Cl} 53.5,53.8))$, followed by IMSS $33.0 \%(95 \% \mathrm{Cl} 32.8,33.1)$.

Table 2 shows CFR for COVID-19 for sex with NCDs. In the total population, the average CFR was $12.1 \%$ (95\% Cl 12.0, 12.2), was higher among men (CFR 14.6\%, 95\% Cl 14.5, 14.8) than women (CFR 9.1\%, $95 \%$ Cl 9.0, 9.3). Trend analyses showed that CFR increased with age and number of NCDs (trend test $p<$ 0.001). In women, the CFR was $1.2 \%$ in the $20-39$-year-old group and $40.8 \%$ in the 80 or older group, while for men it was $2.6 \%$ and $47.9 \%$, respectively.

When comparing the CFR according to the number of NCDs, in the category without NCDs, the CFR in women $(3.4 \%(95 \% \mathrm{Cl} 3.3,3.5))$ and men $(8.0 \%(95 \% \mathrm{Cl} 7.8,8.1))$, across institutions, the highest CFR at IMSS with $10.7 \%(95 \% \mathrm{Cl} 10.5,10.9)$, followed by ISSSTE $(9.5 \%(95 \% \mathrm{Cl} 8.9,10.1))$. In the category $\geq 3$ NCDs, the CFR in women was $(29.9 \%(95 \% \mathrm{Cl} 29.0,30.0))$ and men, $(35.8 \%(95 \% \mathrm{Cl} 34.8,36.7))$, across institutions, IMSS with $44.0 \%(95 \% \mathrm{Cl} 42.9,45.1)$ and ISSSTE $(37.5 \%(95 \% \mathrm{Cl} 35.0,40.1))$. The trend test by number of NCDs were significant $(p<0.001)$ (Appendix 1 , Table 1$)$.

Figure 1 shows that CFR increases with the number of NCDs in a triple interaction $(p<0.01)$ with sex and age. Adults' group 20 to 29 ages with $\geq 3$ NCDs have a greater risk compared to without NCDs, in women $(\mathrm{RR}=46.6,95 \% \mathrm{Cl} 28.2,76.9)$ and men $(\mathrm{RR}=16.5,95 \% \mathrm{Cl} 9.9,27.3)$. Moreover, the risk among adults' group $\geq 80$ ages with $\geq 3$ NCDs compared to without NCDs, in women $1.2(95 \% \mathrm{Cl} 1.0,1.3)$ and men 1.0 $(95 \% \mathrm{Cl} 0.9,1.1)$. The model showed in appendix 2 table 1.

The CFR for COVID-19 in men and women by number and all possible combinations of NCDs is shown in Table 3. For two NCDs, the combination with the greatest CFR was T2D + CKD (CFR $=44.0,95 \% \mathrm{Cl} 39.2$, 48.8); for three NCDs, T2D + COPD + CVD (CFR = 57.5, 95\% Cl 38.4, 75.8). 
When categorizing by number of NCDs (from none to 6), and disaggregating by age group, a greater risk of mortality for older age was found in all categories, except for T2D + CODP and CVD + CKD combinations (Fig. 2). We did not see a specific pattern by sex (Appendix 3, Figs. 1 and 2).

Table 4, Figs. 3, 4 and 5 shows the CFR by age groups and for every disease (T2D, HBP, OB, CKD, COPD and CVD) and the presence of other NCDs. Figure $3 a$ and $3 b$, show in younger adults, the T2D + no other NCDs increases the relative risk in women $(\mathrm{RR}=12.5(95 \% \mathrm{Cl} 7.1,22.2))$ and men $(\mathrm{RR}=5.8(95 \% \mathrm{Cl} 3.1$, 10.9) from no NCDs, the T2D + $1>=$ others NCDs increases the RR in women (RR $=19.9(95 \% \mathrm{Cl} 12.3$, $32.2))$ and in men $(\mathrm{RR}=14.7(95 \% \mathrm{Cl} 10.8,21.6))$ from no NCDs. In contrast with age groups 60 and more, the RR is not more than $70 \%$. This pattern is similarly in all diseases.

\section{Discussion}

In our analysis, the CFR was associated with sex, age and number of NCDs (HBP, obesity, CVD, CKD, or COPD). We observed grater CFR heterogeneity among Institutions.

There is evidence that a country's average age can explain up to $66 \%$ of the variation in CFR for COVID19. ${ }^{21}$ Much of the variation between countries is due to the age of persons evaluated and diagnosed with the virus. In our analysis, the average age of the population infected with COVID-19 was 45 years, similar to China (49 years) but younger than Italy (62 years). ${ }^{2}$ This could be due to the fact that the median age is similar in Mexico (30 years) ${ }^{3}$ and China (35 years), ${ }^{4}$ but lower in Italy (46 years). ${ }^{4}$

Adults with underlying NCDs do not only have a higher probability of getting infected by COVID-19, but also of dying from it ${ }^{22}$ In our analysis, $40.5 \%$ of adults with COVID-19 had at least one NCD. This is greater than the prevalence found in the general Mexican adult population, where $49.2 \%$ have at least one NCD. ${ }^{23}$

COVID-19 is less fatal in women in than men because women's double $X$ chromosome is associated with a greater number of genes that favor immunity. Estrogen modulates the immune response and contributes to the production of more antibodies. ${ }^{7}$ On the other hand, men have less antibodies that decrease the expression of IL-6, which is linked to deregulation of the immune system and lung damage. Likewise, men have higher concentrations of angiotensin-converting enzyme 2 (ACE2) in the alveolar membrane of the lungs through which SARS-CoV-2 enters and infects its host. ${ }^{24}$ Consistent with this infection mechanism and men's diminished immune system response, the CFR in our study was higher in men $(\mathrm{CFR}=14.6)$ than in women $(\mathrm{CFR}=9.1)$.

The number of chronic comorbidities influence the risk of getting infected with COVID-19 and dying from it. ${ }^{25}$ People with more NCDs and of older age have a higher risk of death. ${ }^{10}$ In China, reports indicate that among people $\geq 60$ years, the risk of death was greater among those with two or more NCDs $(R R=2.59)$ in comparison to those with only one NCDs $(R R=1.79) .{ }^{11-16}$ This is consistent with our findings, where 
risk of death was also greater in adults $\geq 60$ years and those with two or more NCDs $(R R=24.1)$ versus those with one $(\mathrm{RR}=14.3)$.

Independent of age, adults with COVID-19 and comorbidities have a greater risk of death. ${ }^{10}$ Adults with COVID-19 and COPD have increased expression of ACE2 in the lower respiratory tract and this explains the greater risk of death. ${ }^{26}$ We observed that among adults with COVID-19 and one NCDs, the highest CFR was found in adults with COPD. When analyzing CFR with two NCDs, the highest rate was found with the combination CKD + T2D. This finding may be due to the fact that hyperglycemia and kidney disease are two of the most common alterations in adults infected by COVID-19, both in Mexico ${ }^{23}$ and Latin America ${ }^{27}$. The common biological mechanism stems from the fact that SARS-CoV-2 expresses ACE2 in greater abundance, which in turn increases the risk of COVID-19 infection, as well as pancreatic ${ }^{28}$ and kidney damage. ${ }^{29}$ The combination of three NCDs with the greatest CFR among Mexicans was CVD + T2D + COPD. This combination is one of the most commonly found in adults around the world ${ }^{27}$ and can be attributed to the fact that multiple organ failure is associated with increased levels of plasminogen, which produces coronary thrombosis and pulmonary embolism. ${ }^{30}$ In general, we found that a greater presence of NCDs increases CFR because it reflects multiorgan dysfunction, severity, and worse prognosis. ${ }^{31-32}$

We observed a triple interaction between number of NCDs, age, and sex. Among adults' group 20 to 29 ages, the presence of NCDs explains the greater probability of death. In this group, the CFR adjusted for women with $\geq 3$ NCDs was $17.2 \%$ versus $0.04 \%$ for without NCDs. This was similar in men with $13.1 \%$ for those with $\geq 3$ NCDs versus $0.8 \%$ for without NCDs. The NCDs explain the largest fraction of CFR in younger adults. In adults' group $\geq 80$ ages, the adjusted CFR for women with $\geq 3$ NCDs was $36.3 \%$ compared to $30.9 \%$ for women without NCDs; for men it was $41.8 \%$, versus $40.1 \%$, respectively. This phenomenon could derive from the prevalence of NCDs, which are present in Mexico from early ages. ${ }^{23-}$ 24

We observed similar associations to other publications that use the same sources, ${ }^{13-14-15}$ however, the present research was an exhaustive analyse with focused on the effect of six NDCs, considering that they have a similar physiopathology, based on expression of angiotensin converting enzyme (ACE2) and a deficient immune system in diabetics that delay the phagocytic and antibacterial activity of neutrophils and macrophages together with oxidative stress that also exacerbates chronic inflammatory processes. ${ }^{33}$ It was decided to include only the variables that observed behaviours congruent with the natural history of NCDs and COVID-19 and the variable of smoking was eliminated since we consider that it does not capture the tobacco habit in a robust way, as proposed by the international recommendations of the experts. ${ }^{34}$

The CFR heterogeneity observed in the Institution where quality care was provided between institutions and the differences in the population served by each. In Mexico, the health institutions can be divided into public and private, in the first can be subdivided into two: a) those that provide care to formal workers, 
whose agencies are the IMSS, the ISSSTE, PEMEX, the SEDENA, and the SEMAR b) those that offer assistance to unemployed people or informal workers, whose units belong to the federal and state health secretariats. With respect to private institutions, these are all health services offered by individuals (with or without health insurance). However, this phenomenon should be studied in detail. ${ }^{35}$

\section{Limitations}

We acknowledge that the main limitation of our study lies within its design, considering it is an observational study. This impedes us from making precise inferences or assuming causal relationships. We lacked information on clinical biomarkers at the time of registration to evaluate the baseline health status of the patients with COVID-19. With this information, we could have estimated, with less error and/or confounders, the effect of NCDs on mortality from COVID-19.

Another possible limitation is due to the data source, which was compiled through the Ministry of Health Epidemiologic Surveillance System and favors surveillance of high risk cases or specific risk factors. This increases the probability of registering cases with severe symptoms and under-representing cases with lower risk who have moderate symptoms.

A third limitation of this study is that, to preserve the confidentiality of participants, the database does not include the specific medical unit in which the patients received care. It did not allow us to control for the specific effect of each health care unit and the clustering of observations at the health care unit, what may affect mainly the variance of our estimates.

A strength of our study is that it is the first to examine the association between CFR and NCDs in COVID19 patients by correcting for the institution of care in all data of Mexican adults registered as COVID-19 positive. Our findings are consistent with other publications that state that NCDs increase mortality in COVID-19 cases. It therefore, contributes to a better understanding of the interaction between COVID-19 and pre-existing NCDs.

\section{Conclusion}

We found that among Mexican adults, mortality from COVID-19 increases with the number of NCDs and this explain the higher mortality in younger adults. Our findings are consistent with the scientific literature and contribute to the understanding of these associations. Is necessary to more studies to understand the heterogenic of mortality by Health Institutions observed. The evidence generated by this study can be useful for decision makers in the health sector, both at the population and clinical level.

\section{Abbreviations}

Case Fatality Rate: CFR; non-communicable diseases: NCDs; high blood pressure: HBP; type 2 diabetes: T2D, cardiovascular disease: CVD; and chronic obstructive pulmonary disease: COPD; obesity: OB; chronic kidney failure: CKD. 


\section{Declarations}

\section{Ethical considerations}

The study does not require ethical review because it is based on open, anonymized data from the Mexican Ministry of Health.

\section{Consent for publication}

Not applicable

\section{Availability of data and materials}

Original data base are public in https://www.gob.mx/salud/documentos/datos-abiertos-152127

\section{Competing interests}

The authors declare that they have no competing interests.

\section{Funding}

It did not have any fund for the investigation.

\section{Author's contribution}

EMF, MRV, SBC and ICN conceptualized the research; EMF and BH analyzed the data; MRV, JEM and ICN Investigation; MRV, JEM, ICN and EMF and ICN methodology; MRV, JEM ICN and EMF supervision; EMF, MRV, JEM, BH, SBC, VEVD, and ICN writing, review and editing.

\section{Acknowledgements}

Not applicable' for that section

\section{References}

1. Coronaviridae Study Group of the International Committee on Taxonomy of Viruses. The Species Severe Acute Respiratory Syndrome-Related Coronavirus: Classifying 2019-nCoV and Naming It SARS-CoV-2. Nat Microbiol. 2020;5(4):36-544.doi: 10.1038/s41564-020-0695-z

2. Chen N, Zhou M, Dong X, Qu J, Gong F, Han Y, et al. Epidemiological and clinical characteristics of 99 cases of 2019 novel coronavirus pneumonia in Wuhan, China: a descriptive study. Lancet. 2020;395(10223):507-13.10.1016/S0140-6736(20)30211-7

3. Wang D, Hu B, Hu C, Zhu F, Liu X, Zhang J, et al. Clinical Characteristics of 138 Hospitalized Patients With 2019 Novel Coronavirus-Infected Pneumonia in Wuhan, China. JAMA. 2020.10.1001/jama.2020.1585 
4. Johns Hopkins University. Mortality analysis EE. UU.: Johns Hopkins University; 2020 [Available from: https://coronavirus.jhu.edu/data/mortality.

5. WHO. Coronavirus disease outbreak (COVID-19) Geneva: World Health Organization; 2020 [Available from: https://www.who.int/es/emergencies/diseases/novel-coronavirus-2019/advice-for-public/q-acoronaviruses.

6. Zhou F, Yu T, Du R, Fan G, Liu Y, Liu Z, et al. Clinical course and risk factors for mortality of adult inpatients with COVID-19 in Wuhan, China: a retrospective cohort study. Lancet. 2020;395(10229):1054-62.10.1016/S0140-6736(20)30566-3

7. Rod JE, Oviedo-Trespalacios 0 , Cortes-Ramirez J. A brief-review of the risk factors for covid-19 severity. Rev Saude Publica. 2020;54:60.10.11606/s1518-8787.2020054002481

8. Wu Z, McGoogan JM. Characteristics of and Important Lessons From the Coronavirus Disease 2019 (COVID-19) Outbreak in China: Summary of a Report of 72314 Cases From the Chinese Center for Disease Control and Prevention. JAMA. 2020.10.1001/jama.2020.2648

9. Li X, Xu S, Yu M, Wang K, Tao Y, Zhou Y, et al. Risk factors for severity and mortality in adult COVID19 inpatients in Wuhan. J Allergy Clin Immunol. 2020.10.1016/j.jaci.2020.04.006

10. Zhang J, Wang X, Jia X, Li J, Hu K, Chen G, et al. Risk factors for disease severity, unimprovement, and mortality in COVID-19 patients in Wuhan, China. Clin Microbiol Infect. 2020;26(6):767-

72.10.1016/j.cmi.2020.04.012

11. Zheng Z, Peng F, Xu B, Zhao J, Liu H, Peng J, et al. Risk factors of critical \& mortal COVID-19 cases: A systematic literature review and meta-analysis. J Infect. 2020.10.1016/j.jinf.2020.04.021

12. Omar Yaxmehen Bello-Chavolla, Jessica Paola Bahena-López, Neftali Eduardo Antonio-Villa. The Journal of Clinical Endocrinology \& Metabolism. 2020;105(8): 2752-2761, /doi.org /10.1210/clinem/dgaa346

13. Denova-Gutiérrez E, Lopez-Gatell H, Alomia-Zegarra JL, et al. The Association of Obesity, Type 2 Diabetes, and Hypertension with Severe Coronavirus Disease 2019 on Admission Among Mexican Patients. 2020; Obesity 2020; 10.1002/oby.22946. doi:10.1002/oby.22946

14. Bello-Chavolla OY, Bahena-Lopez JP, Antonio-Villa NE, Vargas-Vazquez A, Gonzalez-Diaz A, MarquezSalinas A, et al. Predicting Mortality Due to SARS-CoV-2: A Mechanistic Score Relating Obesity and Diabetes to COVID-19 Outcomes in Mexico. J Clin Endocrinol Metab. 2020;105(8).10.1210/clinem/dgaa346

15. Campos-Nonato I, Hernandez-Barrera L, Pedroza-Tobias A, Medina C, Barquera S. Hypertension in Mexican adults: prevalence, diagnosis and type of treatment. Ensanut MC 2016. Salud Publica Mex. 2018;60(3):233-43.10.21149/8813

16. Campos-Nonato I, Ramirez-Villalobos M, Flores-Coria A, Valdez A, Monterrubio-Flores E. Prevalence of previously diagnosed diabetes and glycemic control strategies in Mexican adults: ENSANUT-2016. PLoS One. 2020;15(4):e0230752.10.1371/journal.pone.0230752

17. WHO. Noncommunicable Diseases (NCD) Country Profiles. Mexico. Geneva: World Health Organization; 2018.https://www.who.int/nmh/countries/mex_en.pdf 
18. Secretaria de Salud. Lineamiento estandarizado para la vigilancia epidemiológica y por laboratorio de la enfermedad respiratoria viral. Dirección General de Epidemiología : SSA; 2020 [Available from: http://www.gob.mx/salud.

19. World Health Organization. Laboratory testing of human suspected cases of novel coronavirus (nCoV) infection - Interim guidance. Berlin: WHO; 2020. Contract No.: July

15.https://www.who.int/health-topics/coronavirus/laboratory-diagnostics-for-novelcoronavirus

20. Sudharsanan N, Didzun O, Barnighausen T, Geldsetzer P. The Contribution of the Age Distribution of Cases to COVID-19 Case Fatality Across Countries: A 9-Country Demographic Study. Ann Intern Med. 2020.10.7326/M20-2973

21. Haybar H, Kazemnia K, Rahim F. Underlying Chronic Disease and COVID-19 Infection: A State-of-theArtReview. J Chronic Dis Care. 2020(4):e103452.10.5812/jjcdc.103452

22. Shamah-Levy T, Cuevas-Nasu L, Romero-Martínez M, Rivera-Dommarco J. Encuesta Nacional de Salud y Nutrición 2018. Resultados Nacionales. Cuernavaca, México: Instituto Nacional de Salud Pública; 2020.

23. Jin JM, Bai P, He W, Wu F, Liu XF, Han DM, et al. Gender Differences in Patients With COVID-19: Focus on Severity and Mortality. Front Public Health. 2020;8:152.10.3389/fpubh.2020.00152

24. Martini N, Piccinni C, Pedrini A, Maggioni A. CoViD-19 and chronic diseases: current knowledge, future steps and the MaCroScopio project. Recenti Prog Med. 2020;111(4):198201.10.1701/3347.33180

25. Leung JM, Yang CX, Tam A, Shaipanich T, Hackett TL, Singhera GK, et al. ACE-2 expression in the small airway epithelia of smokers and COPD patients: implications for COVID-19. Eur Respir J. 2020;55(5).10.1183/13993003.00688-2020

26. Clark A, Jit M, Warren-Gash C, Guthrie B, Wang HHX, Mercer SW, et al. Global, regional, and national estimates of the population at increased risk of severe COVID-19 due to underlying health conditions in 2020: a modelling study. Lancet Glob Health. 2020;8(8):e1003-e17.10.1016/S2214109X(20)30264-3

27. Hussain A, Bhowmik B, do Vale Moreira NC. COVID-19 and diabetes: Knowledge in progress. Diabetes Res Clin Pract. 2020;162:108142.10.1016/j.diabres.2020.108142

28. Cheng $Y$, Luo R, Wang $K$, Zhang M, Wang Z, Dong L, et al. Kidney disease is associated with inhospital death of patients with COVID-19. Kidney Int. 2020;97(5):829-38.10.1016/j.kint.2020.03.005

29. Ji HL, Zhao R, Matalon S, Matthay MA. Elevated Plasmin(ogen) as a Common Risk Factor for COVID19 Susceptibility. Physiol Rev. 2020;100(3):1065-75.10.1152/physrev.00013.2020

30. Xu J, Yang X, Yang L, Zou X, Wang Y, Wu Y, et al. Clinical course and predictors of 60-day mortality in 239 critically ill patients with COVID-19: a multicenter retrospective study from Wuhan, China. Crit Care. 2020;24(1):394.10.1186/s13054-020-03098-9

31. Murthy S, Gomersall CD, Fowler RA. Care for Critically III Patients With COVID-19. JAMA. 2020.10.1001/jama.2020.3633 
32. Hodgson K, Morris J, Bridson T, Govan B, Rush C, Ketheesan N. Immunological mechanisms contributing to the double burden of diabetes and intracellular bacterial infections. Immunology. 2015;144(2):171-185. doi:10.1111/imm.12394

33. Grupo de Colaboración de la Encuesta Mundial de Tabaquismo en Adultos. Preguntas sobre el tabaco destinadas a encuestas: Serie de preguntas básicas de la Encuesta Mundial sobre Tabaquismo en Adultos (GATS), $2^{\text {a }}$ edición. Atlanta, GA: Centros para el Control y la Prevención de Enfermedades de los Estados Unidos de América, 2011.

34. Gómez-Dantes,Sesma S, Becerril VM, Knaul FM, Arreola H, Frenk J.The health system of Mexico. Salud Publica Mex 2011;53 suppl 2:S220-S232.

\section{Tables}

Due to technical limitations, tables 1-4 are only available as downloads in the Supplementary Files section.

\section{Figures}




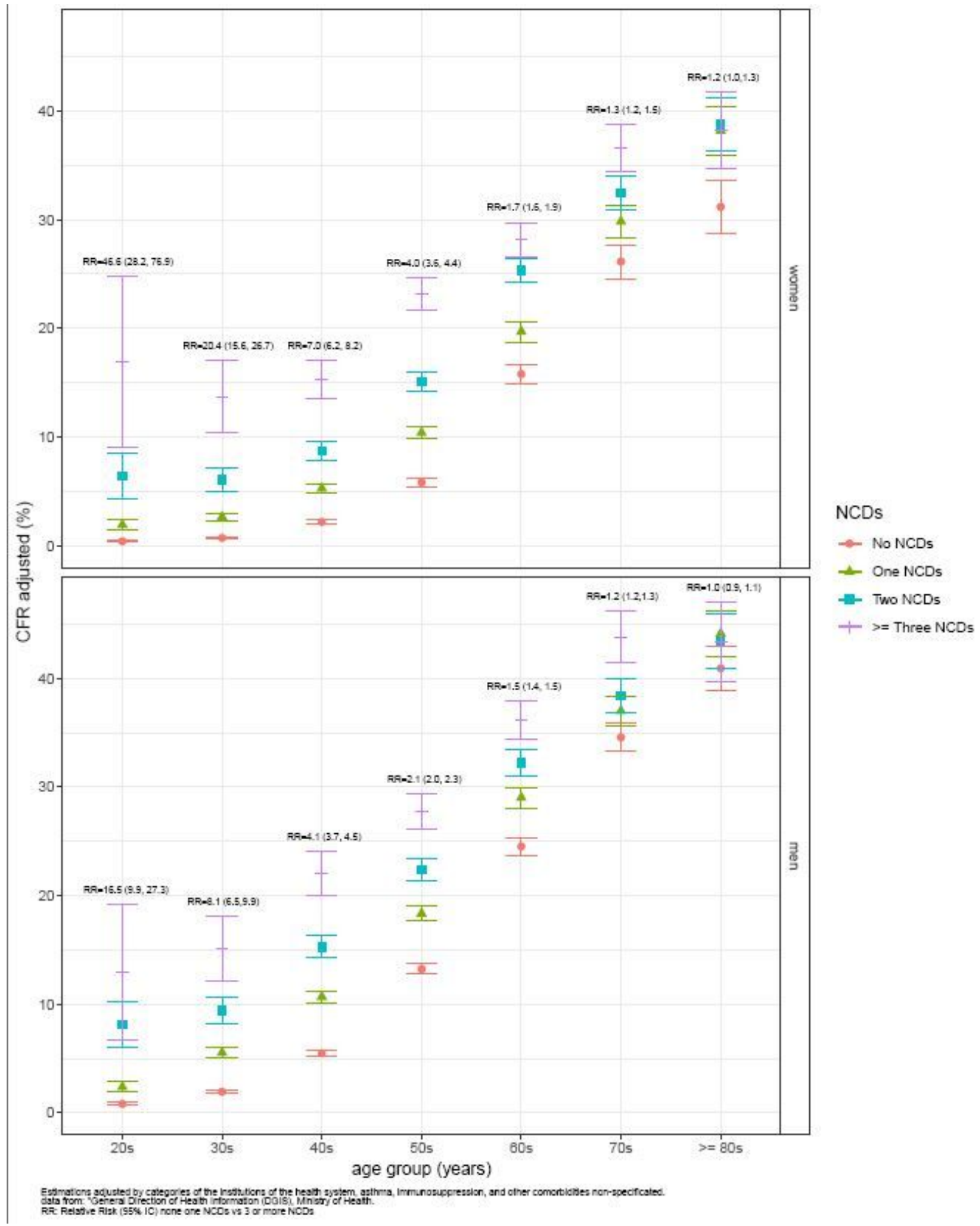

\section{Figure 1}

Case fatality rate (CFR) estimate in adults with COVID-19 and number of non-communicable diseases (NCDs), categorized by age groups and sex. DGIS*, Mexico 2020. 


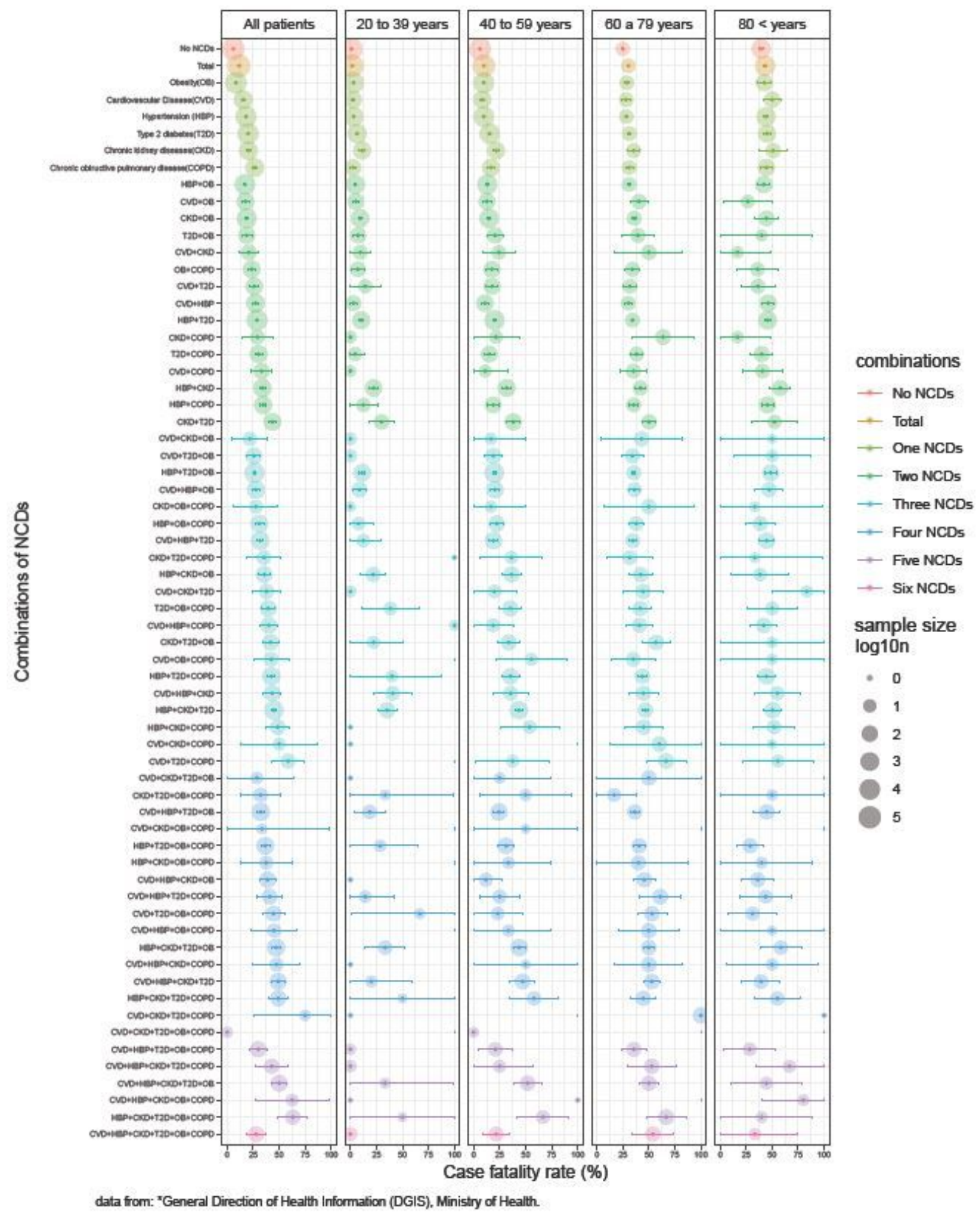

Figure 2

Case fatality rate and combinations of non-communicable diseases (NCDs) by age groups. DGIS*, Mexico 2020. 


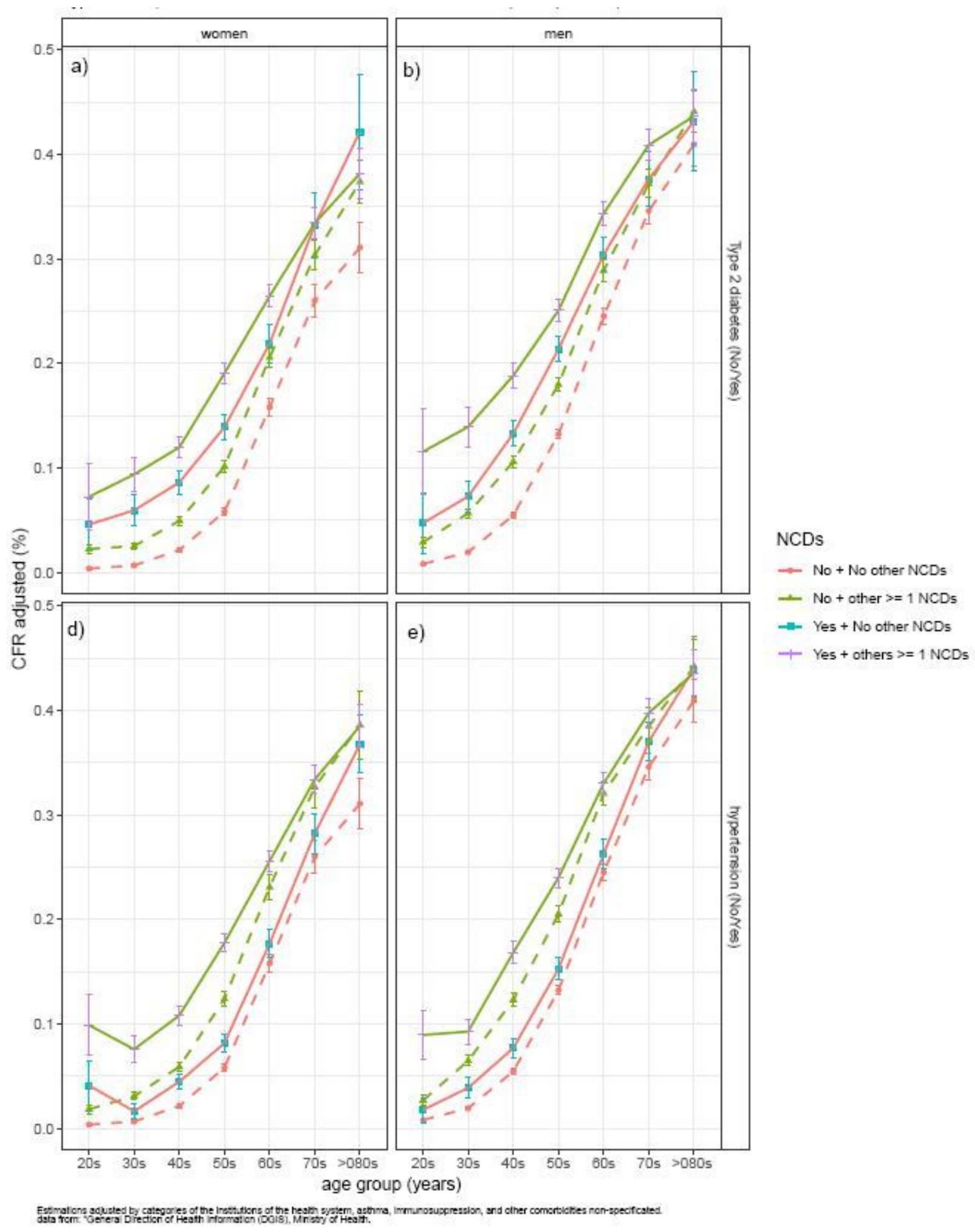

Figure 3

Case fatality rate (CFR) in adults with COVID-19 with/without diabetes or hypertension, with/without other non-communicable diseases (NCDs). DGIS*, Mexico 2020. 


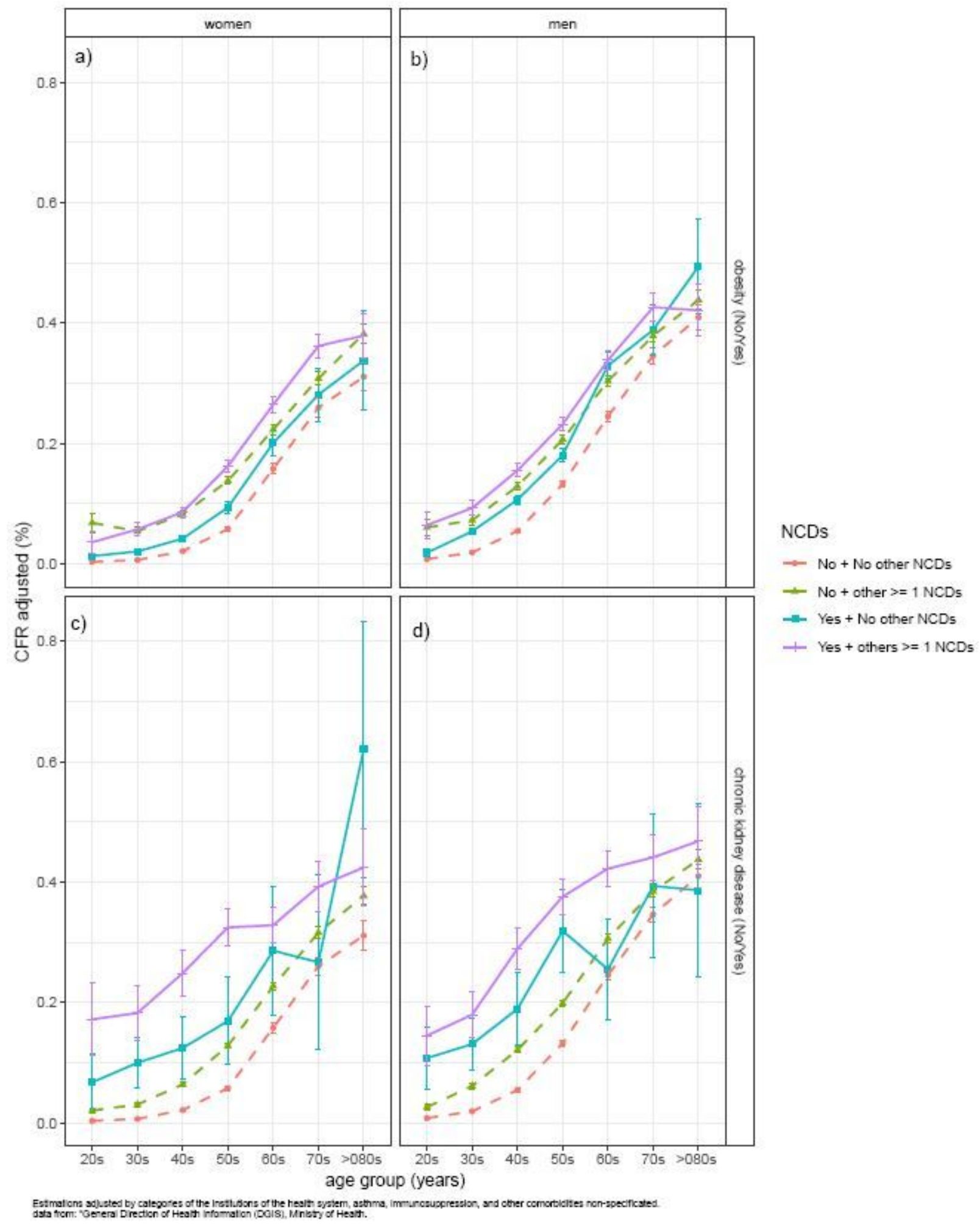

\section{Figure 4}

Case fatality rate (CFR) in adults with COVID-19 with/without obesity or chronic kidney disease, with/without other non-communicable deseases (NCDs). DGIS*, Mexico 2020. 


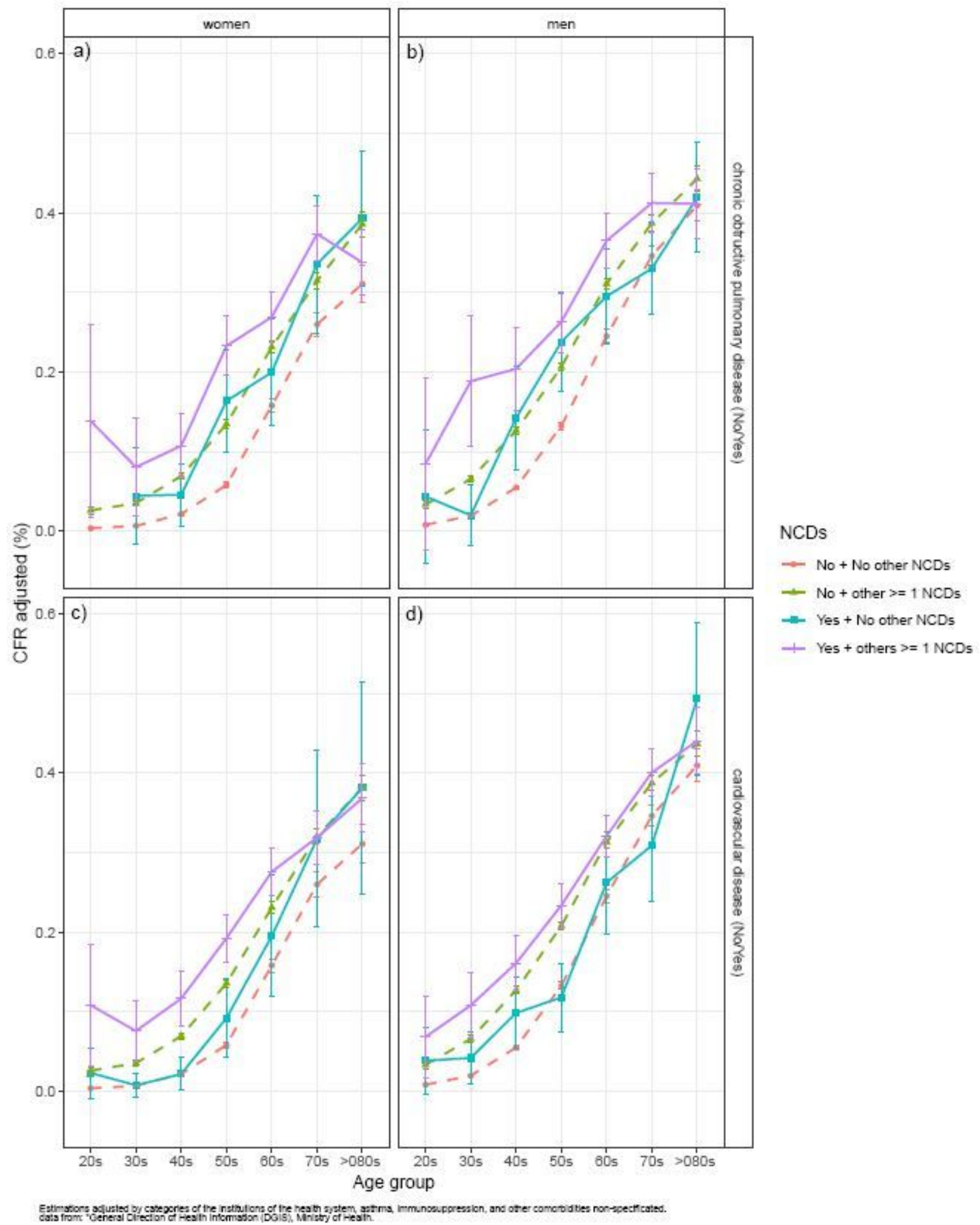

Figure 5

Case fatality rate (CFR) in adults with COVID-19 with/without chronic obstructive pulmonary disease or cardiovascular disease, with/without other non-communicable diseases (NCDs). DGIS*, Mexico 2020.

\section{Supplementary Files}


This is a list of supplementary files associated with this preprint. Click to download.

- supplement1.docx

- supplement2.pdf

- supplement3.pdf

- supplement4.pdf

- supplement5.xIsx

- supplement6.xlsx

- supplement7.xIsx

- supplement8.xlsx 